\title{
Equipment
}

\section{Measurement of pleural pressure with oesophageal catheter-tip micromanometer in anaesthetized humans}

In spontaneously breathing anaesthetized subjects, we studied a new rechnique for the measurement of changes in pleural pressure. Seven ASA physical status I patients undergoing general anaesthesia (enflurane I-2 MAC, nitrous oxide 50\%, and oxygen) for minor orthopaedic surgery were studied in the supine position. Changes in oesophageal pressure ( $\triangle P e s)$ were measured by means of a catheter-tip pressure transducer. This micromanometer was positioned according to an occlusion test where $\Delta P$ es were compared with the changes in airway opening pressure ( $\triangle \mathrm{Pao})$. Optimizing the signalinoise ratio, we observed a linear relationship between $\triangle P$ es and $\triangle P a o$. In each patient, the $\Delta P e s / \Delta P a o$ relationship was highly reproducible. Using the linear regression analysis to characterize the $\Delta P e s / \Delta P a o$ relationship, we have developed a different approach for the positioning of oesophageal catheter. After statistical analysis of the observed $\triangle P e s / \Delta$ Pao relationship, a "calibration" factor can be used in order 10 correct the observed slope of the $\triangle P e s / \triangle P a o$ relationship to its theoretical value. We conclude that an oesophageal catheter-tip micromanometer can be used in anaesthetized supine patients to measure changes in pleural pressure.

\section{Key words}

LUNG: pleural pressure;

MEASUREMENT TECHNIQUES: occlusion pressure, pleural pressure;

EQUIPMENT: catheter-tip pressure transducer; POSITION: supine.

From the Department of Anaesthesia, Hôtel-Dieu de Montréal, University of Montreal.

Address correspondence to: Dr. Daniel Chartrand, Department of Anaesthesia, Hôtel-Dieu de Montréal, 3840 St-Urbain street, Montreal (Quebec), H2W IT8.

Accepted for publication 18th January 1991.
Daniel A. Chartrand MD PhD, Christian Jodoin, Jacques Couture MD FRCPC
Chez des volontaires anesthésiés el respiramt spontanément, nous avons étudié une nowvelle technique de mesure des variations de pression pleurale. Sept patients normaux (ASA I) devant subir une chirurgie orthopédique mineure sous anesthésie générale (enflurane 1-2 MAC, protoryde d'azote $50 \%$, et oxygène) ont été étudiés en décubitus dorsal. Les variations de pression asophagienne ( $\Delta P e s$ ) élaient mesurées au moyen d' un microcapteur de pression situé à l'extrémité d'un cathéter. Ce micromanomètre était positionné selon un test d'occlusion où les $\triangle P$ Pes sont comparés aux variations de pression à l'ouverture des voies aériennes ( $\triangle P a O)$. En optimalisant le rapport signall bruit, nous avons observé une relation linéaire entre les $\triangle P$ es el les $\triangle P a o$. Chez chacun de nos patients, la relation $\Delta P e s / \Delta P a O$ était hautement reproductible. Utilisant l'analyse de régression linéaire pour caractériser la relation $\triangle P e s / \Delta P a o$, nous avons développé une approche différente pour le positionnement d' un cathéter asophagien. Après analyse statistique de la relation $\triangle P e s / \triangle P a o$ observée, un facteur de "calibration" peut être utilisé pour corriger la pente observée de la relation $\Delta P e s /$ $\triangle P a o$ à sa valeur théorique. Nous concluons donc qu' un micromanomètre assophagien peut être utilisé pour mesurer les variations de pression pleurale chez les patients anesthésiés et placés en décubitus dorsal.

During spontaneous breathing or conventional mechanical ventilation, indirect estimation of changes in pleural pressure $(\Delta \mathrm{Ppl})$ is often made by measuring the changes in oesophageal pressure ( $\triangle \mathrm{Pes}$ ). The classical technique used to validate the measurement of $\Delta P e s$ as a reflection of $\Delta \mathrm{Ppl}$ requires that the subjects perform voluntary static Valsalva and Mueller manoeuvres while keeping the glottis open.' The position of the oesophageal catheter is considered acceptable when there is a good agreement between $\Delta P$ es and the changes in airway opening pressure $(\Delta \mathrm{Pao})$. However, in anaesthetized subjects, we must rely on a different approach. 
Since 1978 , several authors ${ }^{2-5}$ have compared $\Delta \mathrm{Pes}$ and $\triangle \mathrm{Pao}$ during spontaneous inspiratory efforts against a closed airway in awake subjects. In 1983, using this dynamic occlusion test and the standard oesophageal balloon technique in anaesthetized supine subjects, Higgs et al. ${ }^{6}$ demonstrated that $\Delta$ Pes can reliably reflect $\Delta \mathrm{Ppl}$ when the oesophageal balloon is properly positioned. This approach is adequate for studies in spontaneously breathing subjects and during conventional mechanical ventilation.

For the study of respiratory mechanics at high frequencies (up to $50 \mathrm{~Hz}$ ), the use of the standard oesophageal balloon technique would not be possible as the frequency response of the oesophageal balloon is inadequate $(\approx 5$ $\mathrm{Hz}$ ). In anaesthetized supine rabbits, Chartrand et al. ${ }^{7}$ demonstrated that an oesophageal catheter-tip pressure transducer can give adequate estimation of $\Delta \mathrm{Ppl}$ up to at least $50 \mathrm{~Hz}$. In the present study, we wanted to assess the validity of the $\Delta$ Pes measurement in anaesthetized supine humans when an oesophageal catheter-tip micromanometer was used.

\section{Methods}

Seven ASA physical status l subjects undergoing general anaesthesia for elective orthopaedic surgery on the lower limbs were studied. Their average age $( \pm S D)$ was $36.3 \pm 16.0 \mathrm{yr}$. The research protocol was approved by the hospital Ethics Committee, and informed consent was obtained from all subjects. An anaesthetist, not directly involved in the experimental protocol, administered the anaesthesia according to the needs of the patient and he was free to interrupt the protocol at any time.

In all subjects, anaesthesia was induced with thiopentone (4-5 $\mathrm{mg} \cdot \mathrm{kg}^{-1}$ ) and muscle relaxation was obtained with succinylcholine $\left(1 \mathrm{mg} \cdot \mathrm{kg}^{-1}\right)$. Tracheal intubation (Sheridan/HVT tracheal tube, $8.0 \mathrm{~mm}$ ID) was performed after laryngeal topical anaesthesia with lidocaine $\left(\approx 1 \mathrm{mg} \cdot \mathrm{kg}^{-1}\right)$. Anaesthesia was maintained with enflurane $(1.5-3.0 \%)$ in a mixture of $50 \%$ nitrous oxide and oxygen. After stabilization of the anaesthesia, the patients were maintained breathing spontaneously and in the supine position.

A 5-F catheter-tip pressure transducer (Millar SPC350) was inserted through a nostril and passed into the stomach. It was then slowly withdrawn to the oesophagus; this was indicated by the negative pressure swings during inspiration. Compared with the oesophageal balloon technique, the catheter-tip micromanometer has a very small volume-pressure displacement coefficient $(<0.001$ $\mu \mathrm{l} \cdot \mathrm{kPa}^{-1}$ ) and large cardiac artifacts can be observed in the retrocardiac portion of the oesophagus $(1-3 \mathrm{kPa})$. However, pulling the catheter more proximally, one can observe a sudden decrease in the amplitude of these cardiac artifacts. We used this position in all our subjects because the signal/noise ratio was greatly improved and a more proximal position was found to decrease the signal/ noise ratio. The signal/noise ratio was obtained by dividing the amplitude of the $\Delta$ Pes related to the respiratory efforts (signal) by the amplitude of the cardiac artifacts (noise). At end-expiratory volume, the tracheal tube was then occluded manually by snugly inserting into its lumen a piezoresistive pressure transducer (Micro Switch 143PC03D). The Millar transducer measured $\triangle P e s$ and the Micro Switch transducer, $\triangle \mathrm{Pao}$. During the next three to four spontaneous breathing efforts, the $\Delta$ Pes were displayed as a function of the $\Delta \mathrm{Pao}$ on a digital storage oscilloscope (Gould 1604). During this dynamic occlusion test, two criteria were used to assess the proper positioning of the oesophageal micromanometer: (1) the cardiac artifacts had to be small $(\approx 0.2 \mathrm{kPa})$; (2) the $\Delta \mathrm{Pes} / \Delta \mathrm{Pao}$ relationship had to be linear and without hysteresis. The position of the catheter being ascertained, three periods of data acquisition (vide infra) were performed within a ten-minute period.

\section{Dala acquisition}

As the experimental set-up was designed for studies of respiratory mechanics at high frequencies, the frequency response of each component was verified. The frequency response of both pressure transducers had been tested using a loudspeaker driven by a sinewave generator and also using step impulses. The oesophageal catheter-tip pressure transducer and the $P$ ao transducer had a flat frequency response over 250 and $4,000 \mathrm{~Hz}$, respectively. Their output signals were amplified (Hewlett Packard, medium gain amplifier 8802A), displayed on a storage oscilloscope (vide supra), and low-pass filtered (Frequency Devices 902LPF) with a cut-off frequency set at $300 \mathrm{~Hz}$. The data acquisition was performed in real time on an "AT compatible" computer with an A/D converter (Data Translation DT2801 A) sampling at 1,024 Hz. For each signal channel, a total of 8,192 data points was sampled in each period of data acquisition. The frequency response of the entire measuring system was verified using step impulses. The two signals had an identical behaviour in both amplitude and phase up to over $200 \mathrm{~Hz}$.

\section{Data analysis}

For each period of data acquisition, the slope of the $\Delta \mathrm{Pes} / \Delta \mathrm{Pao}$ relationship was determined by linear regression. In order to characterize the $\Delta \mathrm{Pes} / \Delta \mathrm{Pao}$ relationship observed in each subject, the mean slope and its standard deviation, the coefficient of determination, and the percentage of reproducibility of the mean slope were computed. The percentage of reproducibility of the mean slope was calculated as: $100 \%$ - S.E.M. \% (S.E.M.\% being the 


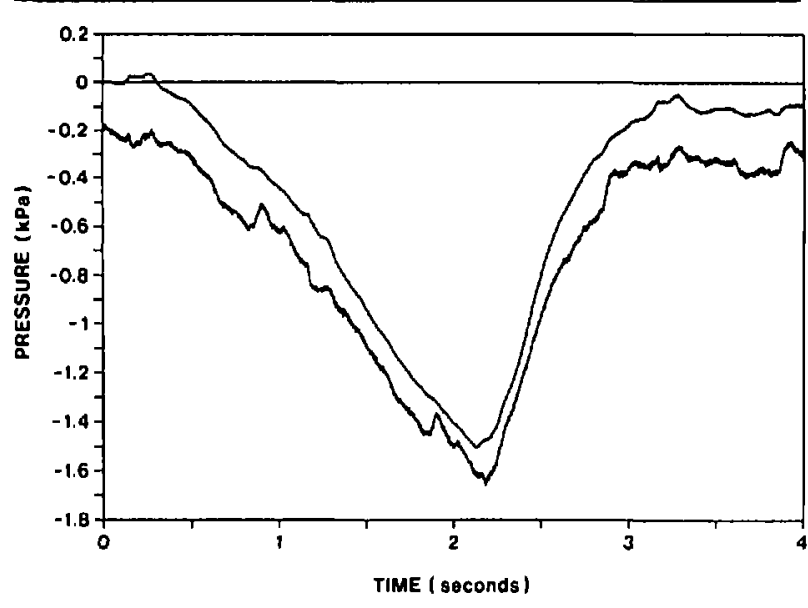

FIGURE 1 Changes of airway opening pressure (upper tracing) and oesophageal pressure (lower tracing) observed during one spontaneous inspiratory effort performed against an occluded tracheal tube in one patient.

standard error of the mean slope expressed as a percentage of that mean value). In order to differentiate respiratory and cardiac components, fast Fourier transforms (FFT) were performed on each signal.

\section{Results}

With the oesophageal micromanometer positioned at the level where the cardiac artifacts decreased suddenly, a linear $\Delta P e s / \Delta P a o$ relationship was observed in all subjects. The distance between the oesophageal micromanometer and the nostril ranged between $281038 \mathrm{~cm}$ and no correlation was found with the height of the patients.

Figure 1 shows the changes in Pao and Pes during a typical occlusion test. Small cardiac artifacts can be observed in the Pes signal (lower tracing). The relationship between $\Delta P e s$ and $\Delta P a o$ during the same occluded inspiratory effort is shown in Figure 2. The mean slope of the $\Delta P e s / \Delta P a o$ relationship observed in each patient (Table) ranged from 0.69 to 1.02 . However, in each individual, the percentage of reproducibility of the mean slope ranged from 97.0 to $99.9 \%$. Furthermore, the variance accounted for by the individual regression lines $\left(r^{2}\right)$ ranged from 92 to $100 \%$ and the average variance not accounted for by the linear regression model was only $\approx 3 \%$. The analysis of the FFT data indicates that the cardiac artifacts were the major contributors to this residual variability.

\section{Discussion}

In previous studies, ${ }^{2-7}$ the measurement of the $\Delta$ Pes was considered to reflect the $\Delta \mathrm{Ppl}$ adequately if the dynamic occlusion test produced a $\Delta \mathrm{Pes} / \Delta \mathrm{Pao}$ relationship with a slope close to 1 . This simple technique is the conventional

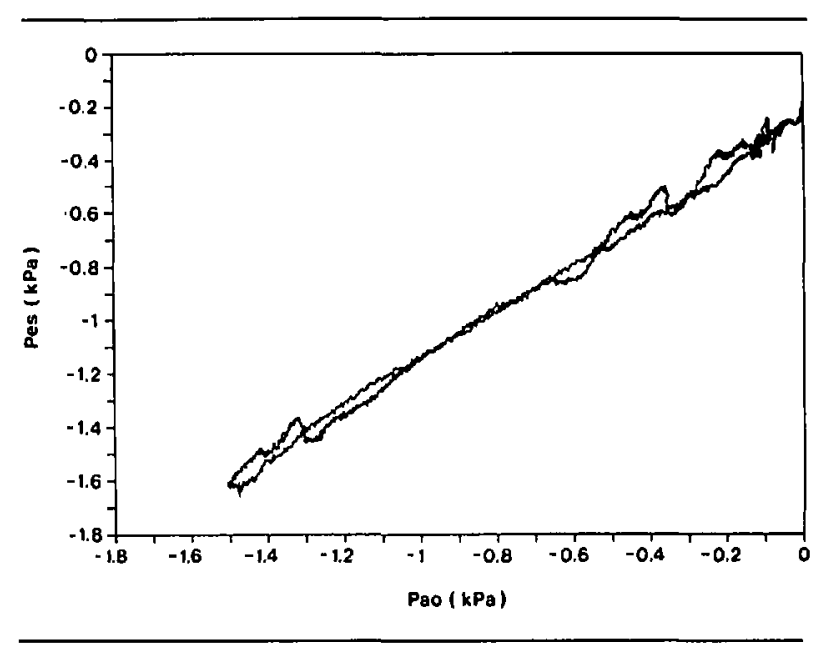

FIGURE $2 \Delta P$ Pes/ $\triangle$ Pao relationship for the occluded inspiratory effort shown in Figure 1. In this patient (\#4), the amplitude of the cardiac artifacts is $=0.1 \mathrm{kPa}$.

method of positioning an oesophageal balloon. However, in the presence of large cardiac artifacts and if the $\Delta \mathrm{Pes} / \Delta \mathrm{Pao}$ relationship is linear and highly reproducible, the most important criterion should be the optimization of the signal/noise ratio. Visualizing the Pes signal while positioning an oesophageal micromanometer (or balloon), one can usually find the quasi-optimal signal/noise ratio. The optimal signal/noise ratio being used to improve the "purity" of the Pes signal, the scattering of the $\Delta$ Pes $/ \Delta$ Pao relationship is minimal and, using linear regression analysis, the highest coefficient of correlation will be obtained. In this study, we observed linear $\Delta P e s / \Delta P a o$ relationships in all the patients and, for each subject, this relationship always had a percentage of reproducibility over $97 \%$. Thus, we focused on improving the signal/ noise ratio.

Using the standard oesophageal balloon technique in anaesthetized supine subjects, Higgs et al. ${ }^{6}$ observed that the mean amplitude of the cardiac artifacts was relatively constant $(\approx 0.2-0.3 \mathrm{kPa})$ at different levels in the oesophagus. However, they mentioned in their discussion: "In

TABLE $\Delta \mathrm{Pes} / \triangle \mathrm{PaO}$ relationships observed in seven patients

\begin{tabular}{llll}
\hline Subject \# & Mean slope $\pm S D^{*}$ & $\%$ of reproducibility & $r^{2}$ \\
\hline 1 & $0.958 \pm 0.004$ & $99.7 \%$ & 0.9859 \\
2 & $0.982 \pm 0.015$ & $99.1 \%$ & 0.9690 \\
3 & $0.923 \pm 0.007$ & $99.5 \%$ & 0.9456 \\
4 & $0.917 \pm 0.002$ & $99.9 \%$ & 0.9959 \\
5 & $1.024 \pm 0.022$ & $98.8 \%$ & 0.9716 \\
6 & $0.691 \pm 0.022$ & $98.2 \%$ & 0.9721 \\
7 & $0.758 \pm 0.039$ & $97.0 \%$ & 0.9464 \\
\hline
\end{tabular}

*SD = standard deviation.

$+r^{2}=$ coefficient of determination. 
our subjects, the optimum balloon positions did not always correspond to the locus of minimum cardiac artifact. The same was true for the conventional balloon position ( $10 \mathrm{~cm}$ from the cardia). Indeed, in the latter position, the cardiac artifact ranged between 4 and $7 \mathrm{~cm}$ $\mathrm{H}_{2} \mathrm{O}(0.4$ and $0.7 \mathrm{kPa})$ in three individuals. In all these subjects, however, by repositioning the balloon, a smaller cardiac artifact could be obtained while maintaining an acceptable $\Delta \mathrm{Pes} / \Delta \mathrm{Pt}$ ratio." Using an oesophageal micromanometer with a very small volume-pressure displacement coefficient, we observed large cardiac artifacts $(1-3 \mathrm{kPa})$ in the retrocardiac portion of the oesophagus. But, pulling the catheter more proximally, we observed a sudden decrease in the amplitude of these artifacts which became similar to those observed by Higgs et al. This reduction of the noise level improved greatly the signal/noise ratio. However, in two subjects (\#6 and \#7), the observed $\triangle P e s / \Delta P a o$ ratio would not be acceptable according to the conventional method of positioning oesophageal catheters.

Studying the $\Delta P e s / \Delta P a o$ relationships at different levels in the oesophagus, Higgs et al. ${ }^{6}$ observed that, in each subject, the optimal slope (close to I) could be found at 5 to $15 \mathrm{~cm}$ from the cardia. Using this conventional method, most investigators will perform an adequate positioning of the oesophageal balloon. Using computerized data acquisition and linear regression analysis, we used a different criterion to select the optimal position of our oesophageal micromanometer: the position where the highest signal/noise ratio and coefficient of correlation can be observed. If, due to thoracic gas decompression during occluded inspiratory efforts, the theoretical $\Delta$ Pes/ $\Delta$ Pao ratio is known to be $\approx 1.02$, it is then possible to correct the slope of the observed $\Delta P e s / \Delta P$ ao relationship if it is highly reproducible. Dividing the theoretical $\Delta \mathrm{Pes} / \Delta \mathrm{Pao}$ ratio by the mean slope of the observed $\Delta P e s / \Delta P a o$ relationships, we obtained a "calibration factor" which could be used to correct the observed $\Delta$ Pes. With our approach, the corrected $\Delta P$ es are not only an "acceptable" ( $\pm 10 \%)$ estimation of the $\Delta \mathrm{Ppl}$ but they are the closest estimation possible ( \pm S.E.M.\%) within the physiological and statistical limits.

As spontaneous breathing is a low-frequency phenomenon, the FFT analyses performed on the Pes and Pao signals did not show harmonics of the respiratory component of the signal over $5 \mathrm{~Hz}$. If the frequency content of the respiratory component of the signal is under $5 \mathrm{~Hz}$, the standard oesophageal balloon technique remains an adequate and inexpensive approach to the measurement of the $\Delta$ Pes. However, the study of respiratory mechanics by high-frequency forced oscillations requires measurement techniques having a much better frequency response. Comparing the FFT analyses performed on simultaneous
Pes and Pao signals, we were able to identify that the frequency content of the cardiac component of the signal was the most important noise in the Pes signal.

We conclude that, in anaesthetized subjects breathing spontaneously in the supine position, the $\Delta \mathrm{Ppl}$ can be estimated from the $\Delta$ Pes measured with an oesophageal micromanometer. However, as the conventional method of positioning oesophageal catheter can not be used in all subjects, we have developed a different approach using the linear regression analysis to characterize the observed $\Delta \mathrm{Pes} / \Delta \mathrm{Pao}$ ratio and correcting this ratio to its theoretical value subsequently.

\section{Acknowledgements}

Dr. Daniel A. Chartrand was the recipient of the 1989 Canadian Anaesthetists' Society Research Award. Christian Jodoin was supported by "Fonds de la recherche en santé du Québec." This work was also supported by "Fondation d'anesthésiologie et réanimation du Québec,"

"Fondation C.O. Monat" (University of Montreal), and "Fondation de l'Hôtel-Dieu de Montréal,"

\section{References}

1 Milic-Emili J, Mead J, Turner JM. Improved technique for estimating pressure from esophageal balloons. J Appl Physiol 1964; 19: 207-11.

2 Asher MI, Coates AL, Collinge JM, Milic-Emili J. Measurement of pleural pressure in neonates. $J$ Appl Physiol 1982; 52: 491-4.

3 Baydur A, Behrakis PK, Zin WA, Jaeger M, Milic-Emili $J$. A simple method for assessing the validity of the esophageal balloon technique. Am Rev Respir Dis 1982; 126: 788-91.

4 Beardsmore CS, Helms P, StocksJ, Hatch DJ, Silverman M. Improved esophageal balloon technique for use in infants. J Appl Physiol 1980; 49: 735-42.

5 Milner AD, Saunders RA, Hopkin LE. Relationships of intraoesophageal pressure to mouth pressure during the measurement of thoracic gas volume in the newborn. Biol Neonate 1978; 33: 314-9.

6 Higgs BD, Behrakis PK, Bevan DR, Milic-Emili J. Measurement of pleural pressure with esophageal balloon in anesthetized humans. Anesthesiology 1983; 59: 340-3.

7 Chartrand DA, Ye TH, Maarek JM, Chang HK. Measurement of pleural pressure at low and high frequencies in normal rabbits. J Appl Physiol 1987; 63: 1142-6. 